\title{
Unusual extra-intestinal manifestations in inflammatory bowel disease (IBD)
}

\author{
G. G. Alroy \\ M.D.
}

A. KURSBAUM
M.D.

\begin{abstract}
Department of Internal Medicine 'B', Rambam Medical Center, and the Aba Khoushy School of Medicine, Haifa, Israel
\end{abstract}

\begin{abstract}
Summary
The extra-intestinal complications of inflammatory bowel disease (IBD) usually appear in patients with long standing active intestinal disease. A patient with Crohn's colitis is described, in whom extra-intestinal symptoms, some of which were unusual, preceded the definitive diagnosis by 9 years.
\end{abstract}

\section{Introduction}

The aetiology of extra-intestinal complications in inflammatory bowel disease (IBD) is unknown. Current thought favours the deposition of circulating antigen-antibody complexes in various tissues (Jewell and MacLennan, 1973). The antigen is believed to be derived from intestinal goblet cells or cross-reacting bacterial antigens (Thayer et al., 1969) and it is thought that active IBD is a pre-requisite for extraintestinal manifestations.

A patient with Crohn's colitis is presented in whom proof of active bowel disease was obtained 9 years after various extra-colonic manifestations were observed.

\section{Report of case}

In September 1967 a 17-year-old male was admitted with a 4-week history of severe pain in the left groin on standing and walking, mild colicky abdominal pain, non-bloody diarrhoea ( 2 or 3 times/day) and low-grade fever. Physical examination was normal except for slight enlargement of the liver and spleen, severe pain on movement of the left hip joint and mild atrophy of the left gluteus muscle.

Laboratory studies revealed an increased ESR (83/89), a mild hypochromic, microcytic anaemia with $11.4 \mathrm{~g} / \mathrm{dl}$ of haemoglobin and a normal leukocyte count; plasma iron of $32 \mu \mathrm{g} / 100 \mathrm{ml}$; stools were positive for occult blood and negative for pathological bacteria and parasites. C-reactive protein, rheumatoid factor, anti-streptolysin titre and LE cells were negative and remained so on subsequent admissions. Liver and kidney function tests and $\mathrm{X}$-ray studies of chest and hip joints were normal. Tests for mal-absorption were negative. Repeated rectosigmoidoscopical examinations were normal except on one occasion when mild congestion of $\stackrel{\vec{\omega}}{\omega}$ the rectal mucosa and a few superficial ulcers wereo noted. Only one of the several biopsies showed 3 . mild plasmocyte and granulocyte mucosal infiltration without ulceration. Repeated barium enema and oj $^{+}$ upper gastro-intestinal series were normal. A diag- $\mathbb{\infty}_{\infty}$ nosis of inflammatory bowel disease with associated $\dot{\infty}$ arthritis prompted the authors to start therapy with $\dot{\circ}$ steroids and sulphasalazine. Under this regimen allo the symptoms rapidly subsided and the patient was $\rightarrow$ discharged.

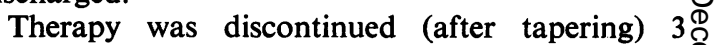
months later. He was well until October 1968, when he was re-admitted because of arthritis of the right hip joint and knee, accompanied by fever. There wege no gastro-intestinal complaints. The outstandia 0 features of the laboratory investigations were a mi thrombocytosis $600 \times 10^{3} / \mathrm{mm}^{2}$ and elevated fibrin $\overline{-}-$ gen of $560 \mathrm{mg} / \mathrm{dl}$.

On the tenth hospital day an acute left ileo-femoral vein thrombosis developed. Thrombectomy was performed on the same day and subsequent heparin $\stackrel{\varnothing}{\Phi}$ therapy instituted.

In December 1968 he developed non-bloody diarrhoea, severe pain in both hip joints, arthritis of $\widetilde{\sigma}$ the temporo-mandibular joints and left wristo joint. X-ray studies of the hips, temporo-mandibular, sacro-iliac and wrist joints were normal as were a 3 barium enema and an upper gastro-intestinalo: series. Recto-sigmoidoscopy was normal. Steroids 3 . and sulphasalazine were given with complete remission of the intestinal and extra-intestinal ${ }^{2}$ manifestations. Over the next 5 years he was followed-o up as an out-patient and remained quiescent on $a_{\supset}$ maintenance dose of sulphasalazine. An ankylosis ofo the left wrist developed (Fig. 1). In March 1974 he presented with fever, dyspnoea and left pleuriticos pain. A pleural friction rub was present posteriorly N $^{\circ}$ at the base of the left lung. Chest X-ray and lung $N_{\mathcal{W}}$ scan were normal. ECG showed inverted $T$ waves ino leads $\mathrm{L}_{3}$ and AVF, consistent with diaphragmatic wall ischaemia. The ESR was $82 \mathrm{~mm}$ in the first hour. Barium enema, recto-sigmoidoscopy, rectalo biopsy and an upper gastro-intestinal series were normal. 


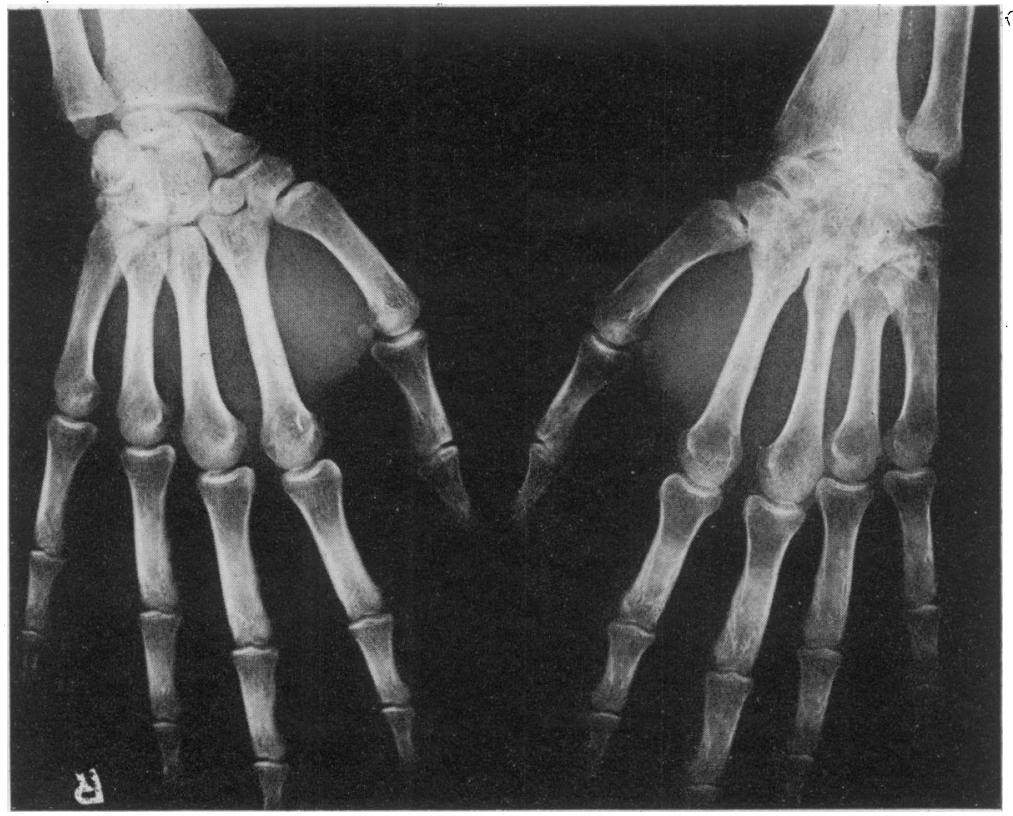

FIG. 1. X-ray showing ankylosis of left wrist,.

On steroid treatment, all the clinical, laboratory and ECG changes reverted to normal. The patient was put on maintenance steroid therapy and remained well until 6 months later when, in January 1975, he was found to have arterial hypertension $(220 / 120 \mathrm{mmHg})$ and microscopic haematuria. For the first time, a barium enema now revealed an abnormal ascending colon with decreased haustral markings, small multiple superficial ulcerations and an oedematous mucosa. The upper gastro-intestinal series was normal. The X-ray appearances were consistent with inflammatory bowel disease (ulcerative colitis or Crohn's colitis) of the ascending colon. The patient was given a diuretic, propranolol and steroids. The blood pressure returned to normal.

During follow-up the antihypertensive therapy was stopped, the steroids tapered and the blood pressure remained normal. In June 1976, he suffered a relapse of diarrhoea, and arthritis of both knees. Discrete violaceous skin lesions that developed into ulcers after scratching were present on the right leg. Barium enema findings were again typical of Crohn's colitis: asymmetrical involvement of the ascending and right transverse colon, decreased haustral markings of the right colon, 'skipped areas' of disease and a thickened ileo-caecal valve. No ulcerations were seen. The upper gastro-intestinal series failed to reveal any small bowel involvement.

\section{Discussion}

The assumption can reasonably be made that the many extra-colonic manifestations in this patient are in fact part of the inflammatory bowel disease. The clinical course, the radiographic features of the large bowel lesion with sparing of the rectum and sigma, indicate a diagnosis of Crohn's disease; however, in the light of the lack of histological proof, those criteria are insufficient for a definite diagnosis (Schachter and Kirsner, 1975). Most extra-intestinal manifestations of IBD appear in patients with clinically, well established, intestinal disease. Occasionally they may precede the intestinal disease by weeks or months but it is indeed rare to observe a latent period of 9 years.

The extra-intestinal complications in this case were unusual and will be discussed system by system.

\section{Joint involvement}

Spondylitis and a sero-negative peripheral arthritis, occur frequently in IBD, Crohn's colitis having the highest incidence (Haslock and Wright, 1973) with decreasing frequency in Crohn's ileitis and ulcerative colitis, in that order. The peripheral arthropathy classically has its onset concurrently with or, generally, after the development of the colitis symptoms. Lower limb joints are affected more often than are the upper. The large rather than the small joints are involved in an asymmetrical fashion. The presence of arthritis correlates very highly with an exacerbation of the underlying bowel disease. The cartilage surface of the joint is not affected and the arthritis generally responds favour- 
ably to medical or surgical treatment of the bowel (Haslock and Wright, 1973).

The joint involvement in this case presented some unusual features in that hip involvement has so far been described only as a septic complication associated with a psoas abscess (Kyle, 1977); and temporomandibular joint involvement and ankylosis of the wrist have, so far, not been described at all.

\section{Arterial and venous thrombosis}

Both are well recognized complications of IBD (Edwards and Truelove, 1964). In post-mortem studies (Bargen and Barker, 1936; Graef et al., 1966) the incidence of thrombosis is invariably higher than appreciated clinically. Various sites of thrombosis have been reported including cerebral, thoracic, epigastric, ileo-femoral and pulmonary veins and temporal artery. Lam et al (1975), in confirming and extending previous reports, have concluded that the hypercoagulable state results from elevated levels of factors V, VIII, fibrinogen and thrombocytes, increased thrombin generation and decreased antithrombin III (due to excessive interaction with thrombin). Bleeding time is normal in those patients and platelets were functionally normal. In all the above mentioned reports there was a tight correlation with exacerbation of the IBD symptoms.

\section{Systemic vasculitis}

This complication was suspected on several occasions in the present patient (pleuritis, electrocardiographic evidence of diaphragmatic ischaemia, transient microhaematuria with hypertension and dermal vasculitis with ulceration). All these lesions resolved with steroid and sulphasalazine therapy. Cases of diffuse vasculitis (Wackers, Tytgat and Vreeken, 1974) and a cutaneous form of polyarteritis nodosa (Solley, Winkelmann and Rovelstad,
1975) have occasionally been described with IBD; $\frac{3}{8}$ myopericarditis is rare (Mowat, 1974) and pul- $\propto$ monary vasculitis has been described in only one $c$ case. An ischaemic ECG pattern, transient nephro- $\overrightarrow{\overrightarrow{\vec{S}}}$ pathy and hypertension as well as pleural involve- $\vec{\sigma}$ ment without parenchymal lung involvement, as $\frac{\bar{\sigma}}{5}$ was observed in the present patient have, so far as $\overline{\bar{c}}$ the authors know, not previously been described.

\section{References}

BARGEN, J.A. \& BARKER, N.W. (1936) Extensive arterial and $\overrightarrow{0}$ venous thrombosis complicating chronic ulcerative colitis. Archives of Internal Medicine, 58, 17.

Edwards, F.C. \& Truelove, S.C. (1964) The course of prognosis of ulcerative colitis. Part III. Complications. Gut, 5, 1.

Graef, V., Baggenstoss, A.H., Sauer, W.G. \& Spittell, 3 . J.A. (1966) Venous thrombosis occurring in non-specific of ulcerative colitis. A necrolepsy study. Archives of Internal Medicine, 117, 377.

HASLOCK, I. \& WRIGHT, V. (1973) The musculo-skeletal complications of Crohn's disease. Medicine, 52, 217.

Jewell, D.P. \& MaCLenNan, I.C.M. (1973) Circulating immunocomplexes in inflammatory bowel disease. Clinical 윽 and Experimental Immunology, 14, 219.

KYLE, J. (1964) Psoas abscess in Crohn's disease. Gastroenterology, 16, 1949.

LAM, A., Borda, I.T., INwood, M.J. \& Thomson, S. (1975) Coagulation studies in ulcerative colitis and Crohn's disease. Gastroenterology, 68, 245.

MowAT, J. (1974) Myopericarditis complicating ulceratike colitis. British Heart Journal, 36, 724.

SCHACHTER, H. \& Kirsner, J.B. (1975) Definitions of in flammatory bowel disease of unknown etiology. Gastrō enterology, 68, 591.

Solley, G.O., WinkelmanN, R.K. \& Rovelstad, R. $\dot{A}$ (1975) Correlation between regional enterocolitis and cutaneous polyarteritis nodosa. Gastroenterology, 69, 235.

Thayer, W.R., Brown, M. \& SANGree, M.H. (1969) Escherichia coli $0: 14$ and colon hemagglutinating anti- $\mathbb{D}$ bodies in inflammatory bowel disease. Gastroenterology, 57, 311.

WACKers, F.J.T.H., Tytgat, G.N. \& VReeken, J. (1974) Necrotizing vasculitis and ulcerative colitis. British Medical Journal, 4, 83. 